\title{
IMBEDDING A REGULAR RING IN A REGULAR RING WITH IDENTITY
}

\author{
NENOSUKE FUNAYAMA \\ Dedicated to the memory of Professor TADAsI Nakayama
}

In [1] L. Fuchs and I. Halperin have proved that a regular ring $R$ is isomorphic to a two-sided ideal of a regular ring with identity. ([1] Theorem 1). Their methed is to imbed the regular ring $R$ in the ring of all pairs $(a, \rho)$ with $a \in R$ and $\rho$ from a suitable commutative regular ring $S$ with identity such that $R$ is an algebra over $S$. Thus $S$ may be seen as the ring of $R-R$ endomorphisms of the additive group of $R$. The following question is naturally raised: Is it true that the ring of all $R-R$ endomorphisms of a rugular ring is a commutative regular ring? The main purpose of this paper is to answer this question affirmatively. (Theorem 1). After established this theorem we can follow the method in [1] to solve the problem in the title.

\section{Endorphisms of $\boldsymbol{R}^{+}$.}

Let $R^{+}$be the additive group of a given ring $R$ with $R$ as left and right operator domains, and let $\widetilde{R}$ be the ring of all endomorphisms of $R^{+}$, that is the ring of all $R-R$ endomorphisms of the additive group $R$. $\widetilde{R}$ has the identity $\overline{1}$ which is the identity mapping of $R^{+}$. Also let us denote by $\overline{0}, \bar{n}$ and $\bar{c}$ respectively the zero endomorphism, $\bar{n}: a \rightarrow n a$, where $\mathrm{a}$ is an element in $R$ and $n$ is an integer, $\bar{c}: a \rightarrow a c$, where $c$ is an element in the center $C$ of $R$.

Lemma 1. If $R$ has the identity 1 , then $\widetilde{R}$ is isomorphic to the center $C$ of $R$.

Proof. Let $\rho$ be an element of $\widetilde{R}$. Then for any element a in $R$ we have $a \rho=(a 1) \rho=a(1 \rho)$ and $a \rho=(1 a) \rho=(1 \rho) a$. Thus $c=1 \rho$ is in the center $C$ of $R$ and $a \rho=a c=c a$. Conversely let $c$ be an element in $C$, then $\bar{c}: a \rightarrow a c$ is an endomorphism of $R^{+}$. $\rho \rightarrow 1 \rho$ sets up a ring isomorphism between $\widetilde{R}$ and $C$.

Lемма 2. If $R^{2}=R$, then $\widetilde{R}$ is commutative.

Received March 22, 1965. 
Proof. Let $\rho, \tau$ be a pair of elements in $\widetilde{R}$. We will show that $a(\rho \tau)=$ $a(\tau \rho)$ for any element $a$ in $R$. As $R^{2}=R$ it is sufficient to show that $(b c)(\rho \tau)$ $=(b c)(\tau \rho)$ for any pair of elements $b, c$ in $R$, and this is easily shown using the fact that $\rho, \tau$ are $R-R$ endomorphisms.

LEMMA 3. If $R$ is a regular ring, then $\widetilde{R}$ is commutative.

Proof is clear by Lemma 2.

For an element $\rho$ in $\widetilde{R}$ denote the kernel and the image of $\rho$ by

$$
\begin{aligned}
& R_{\mathrm{p}}=\rho^{-1}(0)=\{a \in R \mid a \rho=0\}, \\
& \bar{R}_{\mathrm{p}}=\{a \rho \mid a \in R\} .
\end{aligned}
$$

$R_{\mathrm{p}}$ and $\bar{R}_{\mathrm{\rho}}$ are ideals in $R$. If $\rho$ is idempotent then $R=R_{\mathrm{\rho}} \oplus \bar{R}_{\rho}$.

The converse is not always true, that is $R=R_{\mathrm{p}} \oplus \bar{R}_{\mathrm{p}}$ does not imply that $\rho$ is idempotent, and so, for the later use, we seek for the condition for $\rho$ which implies $R=R_{\rho} \oplus \bar{R}_{\rho}$.

LEMMA 4. $R=R_{\mathrm{p}} \oplus \bar{R}_{\mathrm{p}}$ if and only if the following conditions are satisfied:

$$
x \rho^{2}=0 \text { implies } x \rho=0 .
$$

For any $x \in R$ there exists an element $y \in R$ such that

$$
x \rho=y \rho^{2} .
$$

Moreover the $y$ in (2) is uniquely determined in $\bar{R}_{\rho}$.

Proof. Condition (1) is equivalent to the condition $R_{\mathrm{p}} \cap \bar{R}_{\mathrm{p}}=(0)$ as is easily shown. Condition (2) is equivalent to the condition $R=R_{\mathrm{p}}+\bar{R}_{\mathrm{p}}$. Indeed if $R=R_{\mathrm{p}}+\bar{R}_{\rho}$, then any $x \in R$ may be written as $x=x_{1}+x_{2} \rho$, where $x_{1} \rho=0$ and then $x \rho=x_{2} \rho^{2}$. Conversely if the condition (2) is satisfied, any $x \in R$ may be written as $x=(x-y \rho)+y \rho$, where $y$ satisfies $x \rho=y \rho^{2}$. Then $(x-y \rho) \rho=x \rho-y \rho^{2}$ $=0$, which proves that $R=R_{\mathrm{p}}+\bar{R}_{\mathrm{\rho}}$. The proof of the last part is as follows: First the $y$ in (2) may be chosen from $\bar{R}_{\rho}$ as $x \rho=y \rho^{2}$ and $y \rho=y \rho^{2}$ imply that $x \rho=(z \rho) \rho^{2}$. Secondly the uniqueness of $y$ : If $x \rho=y \rho^{2}=z \rho^{2}$, where $y$ and $z$ are in $\bar{R}_{\rho}$, then $(y-z) \rho^{2}=0$, which implies $(y-z) \rho=0$ by (1). As $y$ and $z$ are in $\bar{R}_{\rho} y=y^{\prime} \rho, z=z^{\prime} \rho$ for some $y^{\prime}, z^{\prime} \in R$. Then $\left(y^{\prime}-z^{\prime}\right) \rho^{2}=0$, and so again by (1) $\left(y^{\prime}-z^{\prime}\right) \rho=0$, that is $y=z$.

LEMMA 5. If $\rho \in \widetilde{R}$ satisfies $R=R_{\rho} \oplus \bar{R}_{\rho}$, then for some $\sigma \in \widetilde{R}$,

$$
\rho \sigma \rho=\rho
$$




$$
\begin{aligned}
& \rho \sigma=\sigma \rho \\
& \sigma \rho \sigma=\sigma
\end{aligned}
$$

Proof. In Lemma 4 it is shown that $R=R_{\rho} \oplus \bar{R}_{\mathrm{\rho}}$ implies that, for any $x \in R$ there exists uniquely determined $y \in \bar{R}_{\rho}$ with $x_{\rho}=y \rho^{2}$. Define $\sigma$ as $x_{\sigma}=y$. As is easily seen $\sigma$ is an endomorphism of the additive group of $R$. For any elements $x, r$ in $R$ we have

$$
(x r) \rho=(x \rho) r=\left(y \rho^{2}\right) r=(y r) \rho^{2} .
$$

As $\bar{R}_{\mathrm{p}}$ is an ideal of $R$ we have $y r \in \bar{R}_{\rho}$, showing that $(x r)_{\sigma}=\left(x_{\sigma}\right) r$. Similarly $(r x)_{\sigma}=r\left(x_{\sigma}\right)$. Thus $\sigma \in \widetilde{R}$.

As the proofs of (3), (4) and (5) are similar we show only (5). To prove (5) it is sufficient to show that $x(\sigma \rho \sigma)=x \sigma$ for any $x \in R$. Put $x \sigma=y$ and $x(\sigma \rho \sigma)$ $=z$. Then, by the definition of $\sigma$, we have $x \rho=y \rho^{2}, y \in \bar{R}_{\rho}$, and $(y \rho) \sigma=z$, that is $y \rho^{2}=z \rho^{2}$, where $y$ and $z$ are in $\bar{R}_{\rho}$. Then $(y-z) \rho^{2}=0$, which implies $y=z$ as $y$ and $z$ are in $\bar{R}_{\mathrm{p}}$. Thus we have $x \sigma=x(\sigma \rho \sigma)$.

THEOREM 1. The ring $\widetilde{R}$, ring of all endomorphisms of $R^{+}$, of a regular ring $R$ is a commutative regular ring with identity.

Proof. Commutativity was already shown in Lemma 3. To prove the regularity of $R$ it is sufficient to prove $R=R_{p} \oplus \bar{R}_{p}$ for any $\rho \in \widetilde{R}$, or equivalently, by Lemma 4, (1) and (2) in Lemma 4. Suppose that $x \rho \neq 0$. Then by the regularity of $R$ there exists $y \in R$ such that $x \rho=(x \rho) y(x \rho)$. This implies $x \rho=$ $\left(x \rho^{2}\right) y x$ and as $x \rho \neq 0$ we have that $x \rho^{2} \neq 0$ showing (1). Also $x \rho=(x \rho) y(x \rho)=$ $(x y x) \rho^{2}$ showing (2).

\section{Imbedding a regular ring into a regular ring with identity.}

Let $R$ be an arbitrary ring.

Let $S$ be a commutative subring of $\widetilde{R}$, the ring of all $R-R$ endomorphisms of $R^{+}$, and let $R^{s}$ be the set of all ordered pairs $(a, \rho)$ where $a \in R$ and $\rho \in S$. In $R^{S}$ define the equality, addition, and multiplication by

$$
\begin{aligned}
& (a, \rho)=(b, \tau) \text { if and only if } a=b \text { and } \rho=\tau, \\
& (a, \rho)+(b, \tau)=(a+b, \rho+\tau) \\
& (a, \rho)(b, \tau)=(a b+b \rho+a \tau, \rho \tau) .
\end{aligned}
$$

Then $R^{S}$ is a ring. Commutativity of $S$ is used for the proof of associativity of $R^{s}$. If $S$ has the identity then $R^{s}$ has the identity $(0, \overline{1})$. The examples of 
$S$ are as follows: (a) $Z=\{\bar{n}: a \rightarrow n a, n$ is an integer $\}$, (b) $\bar{C}=\{\bar{c} \mid \bar{c}: a \rightarrow a c$ ( =ca), $c$ is in the center $C$ of $R$, (c) $\bar{Z}+\bar{C}$, (d) $\widetilde{R}$ when $\widetilde{R}$ is commutative.

Remark 1. $R^{\bar{z}}$ does not coincide with the classical imbedding $R^{\#}$. Indeed when $R$ is of bounded order $R^{\bar{z}}$ is of bounded order but $R^{\#}$ is not of bounded order.

$R$ is imbedded in $R^{S}$ as an ideal by the mapping $a \rightarrow(a, 0)$. Our idea is to give some properties to $R^{s}$ selecting a suitable $S$. This idea is essentially included in [1], and the proof of the following theorem follows that in [1].

Lемма 6. If $R$ and $S$ are regular, then $R^{s}$ is regular.

Proof. Let $(a, \rho)$ be any element in $R^{s}$. We will seek for $(b, \sigma)$ such that $(a, \rho)(b, \sigma)(a, \rho)=(a, \rho)$, that is

$$
\begin{aligned}
& \rho \sigma \rho=\rho, \\
& a b a+(b a) \rho+(a b) \rho+a^{2} \sigma+b \rho^{2}+a(\sigma \rho)+a(\rho \sigma)=a .
\end{aligned}
$$

As $S$ is regular there exists a $\sigma$ such that $\rho \sigma \rho=\rho$. For the second equality: Let $\boldsymbol{e}$ be an idempotent in $R$ such that $a=a \boldsymbol{e}=\boldsymbol{e}$. (The existence such an $\boldsymbol{e}$ has been proved in [1] Lemma 2).

By the regularity of $R$ there exists an element $x$ such that

$$
(a+e \rho) x(a+e \rho)=a+e \rho .
$$

Put $y=e x e$, then, as is easily calculated, $y$ satisfies (7) replacing $x$ by $y$. Put $b=y-e_{\sigma}$, then $b$ satisfies (6).

THEOREM 2. $R^{\tilde{R}}$ is a regular ring with identity if $R$ is regular. $R$ is imbedded in $R^{\tilde{R}}$ as an ideal.

Proof is clear from Theorem 1 and Lemma 6.

\section{REFERENCE}

[1] L. Fuchs and I. Halparin, On the embedding of a regular ring in a regular ring with identity, Fundamenta Mathematicae LIV (1964), pp. 287-290.

Yamagata University 